\title{
RELACIÓN ENTRE LA PRÁCTICA DE LA ACTIVIDAD FÍSICA-DEPORTIVA Y LA MEJORA DEL BIENESTAR FÍSICO-PSICOLÓGICO EN PERSONAS MAYORES
}

\author{
RELATION BETWEEN THE PRACTICE OF THE PHYSICAL-SPORTS ACTIVITY AND \\ THE IMPROVEMENT OF THE PHYSICAL-PSYCHOLOGICAL WELL-BEING IN MAJOR PERSONS
}

Manuel Isorna Folgar*

Marta Felpeto Lamas**

Resumen

\begin{abstract}
La clave para contar con una población sana es el denominado por la Organización Mundial de la Salud envejecimiento activo, el cual incluye estrategias de promoción de salud y prevención de las discapacidades e involucra programas de ejercicio físico o actividad física practicados de manera regular. Cada vez toma más auge el hecho de que la realización de una vida activa, con práctica de actividades y ejercicios físicos, ayuda a prevenir la aparición de enfermedades y mejora el bienestar subjetivo. Por ello, el objetivo del presente estudio es realizar una revisión de las ventajas de la práctica físico-deportiva en personas adultas, así como realizar una serie de recomendaciones para aumentar la eficiencia y eficacia de su puesta en práctica.
\end{abstract}

Palabras clave: actividad física, personas adultas, mayores, envejecimiento.

\section{Abstract}

The key to having a healthy population is called by the World Health Organization "Active Ageing", which includes strategies for health promotion and prevention of disabilities and involves physical exercise programs or regularly physical activity practices. An essential growth takes the fact that the realization of an active lifestyle, with practical activities and exercise, helps to prevent the onset of diseases and improves subjective well-being. Therefore, the objective of this study is to conduct a review of the benefits of physical sports in adults as well as making a number of recommendations to increase the efficiency and effectiveness of its implementation.

Keywords: physical activity, adults, seniors, aging.

Fecha de recepción: 12 de diciembre de 2013

Fecha de aprobación: 28 de marzo de 2014

\footnotetext{
* Doctor en Psicología, Máster en Drogodependencias, Licenciado en Psicología. Docente de la Universidad de Vigo (España) Facultad de Ciencias de la Educación. Correo electrónico: isorna.catoira@uvigo.es

** Diplomada en Educación Social, Universidad Santiago de Compostela. Correo electrónico: mfelpeto@gmail.com
} 


\section{INTRODUCCIÓN}

En España, al igual que en otros países del mundo, la expectativa de vida está aumentando. Sin embargo, la prolongación de la vida no siempre se acompaña de una buena calidad de esta (tabla 1). La disminución de la actividad y ejercicio físico (estilo de vida sedentario) es un hecho preocupante en todos los países del mundo con economías desarrolladas. A partir de los 25 años, y a medida que las personas van envejeciendo, van realizando menor cantidad e intensidad de actividad física, lo que les encaminará a desarrollar posteriormente determinadas enfermedades con sus respectivas limitaciones, siendo estas, en muchas ocasiones, las razones esgrimidas para justificar la no realización de ejercicio físico dadas la restricciones propias de cada enfermedad y producidos por los problemas crónicos de salud y que se irán acentuando con la edad.

Tabla 1. Esperanza de vida (EV), esperanza de vida con buena salud (EVBS) y años con mala salud (MS) en España.

Fuente: Ministerio de Sanidad y Política social, 2010.

\begin{tabular}{|l|l|l|l|l|l|l|l|}
\hline \multicolumn{1}{|c|}{ COMUNIDAD } & \multicolumn{1}{|c|}{ EV } & \multicolumn{1}{|c|}{ EVBS } & \multicolumn{1}{|c|}{ MS } & COMUNIDAD & EV & EVBS & MS \\
\hline Andalucía & 79.8 & 54.8 & 25 & Extremadura & 80.6 & 52.6 & 28.0 \\
\hline Aragón & 81.7 & 59.1 & 22,6 & Galicia & 81 & 47.8 & 33.2 \\
\hline Asturias & 80.4 & 53.8 & 22.6 & Madrid & 82.5 & 58.6 & 23.9 \\
\hline Baleares & 81.6 & 57.8 & 23.8 & Murcia & 80.5 & 50.7 & 29.8 \\
\hline Canarias & 80.4 & 54.5 & 25.9 & Navarra & 82.5 & 57.6 & 24.9 \\
\hline Cantabria & 81.1 & 59.6 & 21.5 & País Vasco & 81.6 & 59.8 & 21.8 \\
\hline Castilla-La Mancha & 81.5 & 55.2 & 26.3 & La Rioja & 81.8 & 62.8 & 19.0 \\
\hline Castilla y León & 82.1 & 61.3 & 20.8 & Ceuta y Melilla & 79.5 & 52.2 & 26.7 \\
\hline Cataluña & 81.6 & 55.2 & 26.4 & & & & \\
\hline Comunidad Valenciana & 80.6 & 52.5 & 28.1 & Total & 81.1 & 55.3 & 25.8 \\
\hline
\end{tabular}

La buena noticia es que la merma de las habilidades físicas que suceden habitualmente con la edad se puede prevenir, e incluso revertir, a través de una atención apropiada de nuestros niveles de condición y actividad física. Los beneficios del ejercicio regular y la actividad física contribuyen a un estilo de vida más saludable e independiente para los mayores, mejorando ampliamente sus capacidades funcionales y calidad de vida (ACSM, 2001).
Por otro lado, también estamos comprobando cómo la práctica habitual de ejercicio se va extendiendo en mayor o menor medida en las últimas décadas; sobre todo para contribuir a la curación y rehabilitación de diversas dolencias o enfermedades y/o por la prevención de otras; todo ello nos está llevando a observar a muchas personas mayores que se van incorporando poco a poco a la práctica de algún deporte o actividad física. Disponer de mayor tiempo libre tras la jubila- 
ción, la prescripción médica o simplemente la oferta de muchos polideportivos o instalaciones deportivas municipales con programas de ejercicio monitorizados y dirigidos a personas mayores son algunas de las razones por las que este importante grupo de población inicia algún tipo de actividad deportiva. A pesar de todo, el número de personas mayores que realizan ejercicio físico de forma habitual es todavía muy bajo. Según los datos recogidos en España (García Ferrando, 2006), el $70 \%$ de las personas mayores suelen dar paseos y acudir al parque, una proporción similar realiza compras y recados y un $10 \%$ manifiesta realizar deporte en un club o sociedad recreativa. Además, el 39\% de las personas que tienen entre 55-64 años considera que su forma física es buena o excelente, lo que en cierta medida no se corresponde con los datos aportados en la tabla 1. En cuanto a la frecuencia de práctica, el 51\% de las personas de 55-64 años y el 54\% de los mayores de 65 realizan actividad física tres o más días por semana. En lo que respecta a los motivos que llevan a realizar práctica deportiva a los sujetos de edades por encima de los 55 años, destacan hacer ejercicio físico, mantener o mejorar la salud y la diversión, entre otros (Moreno, Martínez, González-Cutre y Cervelló, 2008).

Sin embargo, también nos encontramos con que la sociedad actual no favorece la actividad física, y factores como la automatización de las fábricas, los sistemas de transporte o la amplia gama de equipos electrónicos en las viviendas han reducido de forma muy apreciable la necesidad de desarrollar trabajo físico y han fomentado el sedentarismo (Jackson, Morrow, Hill y Dishman, 2003). El resultado es que la vida se ha tornado mucho más fácil y resulta más complicado encontrar el tiempo y la motivación suficientes para mantener una forma física aceptable. En el futuro se prevé que este fenómeno, si no se toman medidas, será aún más preocupante, y que el desarrollo de la tecnología inalámbrica puede disminuir aún más la práctica de la actividad física. En la industria de las nuevas tecnologías, el movimiento se considera sinónimo de ineficacia y la reducción del tiempo invertido en él es una de las claves del aumento de la productividad; un modelo que, desafortunadamente, se está transmitiendo a los países en desarrollo.

Sea como fuere, el aumento de la esperanza de vida en las sociedades avanzadas como consecuencia del desarrollo económico y el bienestar alcanzado ha ocasio- nado un cambio radical en las pirámides demográficas, produciéndose un aumento de enfermedades no transmisibles, las cuales aumentan el gasto sanitario de los Estados y, por otro, limitan la autonomía, independencia y calidad de vida de las personas mayores (y no tan mayores); es decir, la inactividad física, además de la contribución a la aparición de las enfermedades endocrinas y metabólicas, magnifica los problemas físicofuncionales como la pérdida de fuerza muscular, flexibilidad, equilibrio, vista, memoria y audición, así como en los problemas psicosociales como: sentimiento de pérdida de salud, tristeza, deterioro de las capacidades funcionales y discriminación familiar, entre otros (Jiménez, Núñez y Coto, 2013).

No debemos olvidar que, puesto que es irremediable envejecer, lo que sí podemos cambiar o modificar es la velocidad o incluso la reversibilidad potencial de este proceso. A través del ejercicio y la actividad física regular, podemos contribuir a mantener y desarrollar un estilo de vida más saludable e independiente para nuestros mayores, mejorando ampliamente sus capacidades funcionales y calidad de vida.

\section{MEDIDA DE LA ACTIVIDAD FÍSICA, GASTO ENERGÉTICO Y EXCESO DE PESO}

Es importante, antes de analizar los efectos que presenta la actividad física sobre la salud, establecer qué se entiende por actividad física y por términos relacionados, como ejercicio físico o forma física.

La actividad física se refiere a la energía utilizada para el movimiento. Se trata, por tanto, de un gasto de energía adicional al que necesita el organismo para mantener las funciones vitales tales como respiración, digestión, circulación de la sangre, etc. La contribución fundamental a la actividad física diaria se debe a actividades cotidianas tales como andar, transportar objetos, subir escaleras, hacer las tareas del hogar o ir a la compra.

El término ejercicio hace referencia a movimientos diseñados y planificados específicamente para estar en forma y gozar de buena salud. Aquí se podrían incluir actividades como aeróbicos, ciclismo, andar a paso ligero o jardinería. Si, además, el ejercicio físico se realiza como competición que se rige por unas reglas determinadas, hablamos de deporte. 
La forma física, a diferencia de la actividad física o el ejercicio, que son procesos conductuales, se corresponde con una serie de atributos como fuerza o resistencia que determinan la capacidad para realizar actividad física. La forma física depende tanto de factores genéticos como de los niveles de actividad física de los individuos, de modo que es posible desarrollar programas específicos de ejercicio encaminados a la mejora de la forma física.

La cuantificación del gasto energético asociado a la actividad física se puede realizar en kilocalorías o kilojulios $(1 \mathrm{kcal}=4,20$ kjulios; 1.000 kilojulios $=240$ kilocalorías). Para facilitar la tarea de dicha cuantificación y de medir la intensidad de la actividad física, muchos especialistas utilizan una unidad denominada MET (equivalente metabólico). Un MET es igual al número de calorías que un cuerpo consume mientras está en reposo. A partir de ese estado, los MET se incrementan según la intensidad de la acción.

El metabolismo basal es el valor mínimo de energía necesaria para que la célula subsista. Esta energía mínima es utilizada por la célula en las reacciones químicas intracelulares necesarias para la realización de funciones metabólicas esenciales, como es el caso de la respiración.

La tasa metabólica disminuye con la edad y con la pérdida de masa corporal. El aumento de la masa muscular es lo único que puede incrementar esta tasa. El metabolismo basal de una persona se mide después de haber permanecido en reposo total en un lugar con una temperatura agradable $\left(20^{\circ} \mathrm{C}\right)$ y de haber estado en ayunas doce o más horas.

Según recogen Márquez, Rodríguez y De Abajo (2006), el metabolismo basal diario se puede calcular de manera muy aproximada de la siguiente forma mediante las ecuaciones de Harris Benedict:

- Hombre: $66,4730+((13,751 \times$ masa $(\mathrm{kg}))+$ $(5,0033 \mathrm{x}$ estatura $(\mathrm{cm}))-((6,55 \mathrm{x}$ edad (años $))$

Mujer: $655,1+((9,463 \times$ masa $(\mathrm{kg}))+$
$(1,8 \times$ estatura $(\mathrm{cm}))-((4,6756 \times$ edad $($ años $))$

Debemos tener presente que los siguientes factores aumentan el metabolismo basal: mayor masa muscular, mayor superficie corporal total, sexo masculino (Los varones casi siempre tienen mayor masa corporal magra que las mujeres), temperatura corporal (fiebre o condiciones ambientales frías), hormonas tiroideas (un regulador clave del metabolismo basal), aspectos de la actividad del sistema nervioso (liberación de hormonas de estrés), etapas de crecimiento en el ciclo vital, consumo de cafeína o tabaco (no se recomienda el uso de tabaco para controlar el peso corporal ya que aumenta demasiado los riesgos a la salud).

A continuación (tabla 2) se detallan los MET de algunas actividades cotidianas, laborales y físicas que pueden servir de guía para determinar cuál es nuestro gasto energético aproximado durante el día.

Tabla 2. Ejemplos de cuantificación de las actividades mediante MET.

Fuente: adaptado de Márquez et al., 2006.

\begin{tabular}{|l|l|l|l|}
\hline \multicolumn{1}{|c|}{ Intensidad } & \multicolumn{1}{|c|}{$\begin{array}{c}\text { Actividades en } \\
\text { el hogar }\end{array}$} & \multicolumn{1}{c|}{ Actividades laborales } & \multicolumn{1}{c|}{ Actividad física } \\
\hline Muy Liviana (3 MET) & $\begin{array}{l}\text { Cocinar, vestirse, } \\
\text { ducharse... }\end{array}$ & $\begin{array}{l}\text { Trabajar en el computador, tra- } \\
\text { bajos de permanencia senta- } \\
\text { dos (cajeros, administración...) }\end{array}$ & $\begin{array}{l}\text { Caminar lento en } \\
\text { un sitio plano. }\end{array}$ \\
\hline Liviana (3 a 5 MET) & $\begin{array}{l}\text { Limpiar ventanas, pasar } \\
\text { aspiradora, barrer, } \\
\text { ordenar la casa... }\end{array}$ & $\begin{array}{l}\text { Trabajos manuales tipo arre- } \\
\text { glar desperfectos... }\end{array}$ & $\begin{array}{l}\text { Caminar con marcha } \\
\text { ligera, andar en bici- } \\
\text { cleta e sitio plano... }\end{array}$ \\
\hline Pesada (6 a 9 MET) & $\begin{array}{l}\text { Subir escaleras, car- } \\
\text { gar las bolsas de } \\
\text { la compra... }\end{array}$ & $\begin{array}{l}\text { Realizar trabajos de albañilería, } \\
\text { o con instrumentos pesados... }\end{array}$ & $\begin{array}{l}\text { Jugar al fútbol, esquí } \\
\text { de fondo, patinar, } \\
\text { correr suave, pasear } \\
\text { en piragua... }\end{array}$ \\
\hline $\begin{array}{l}\text { Muy pesada } \\
\text { (superior a 9 MET) }\end{array}$ & $\begin{array}{l}\text { Subir escaleras } \\
\text { rápido, levantar bol- } \\
\text { sas muy pesadas. }\end{array}$ & $\begin{array}{l}\text { Cortar leña, mover cajas } \\
\text { muy pesadas... }\end{array}$ & $\begin{array}{l}\text { Jugar al rugby, } \\
\text { correr rápido en } \\
\text { campo a través... }\end{array}$ \\
\hline
\end{tabular}


Para convertir 1 MET en Kcal./min., aplicamos la siguiente ecuación:

Kcal./min.=MET x 0,0175 x PESO (Kg.)

Ejemplo: si pesas $60 \mathrm{Kg}$. y corres a $8 \mathrm{Km} / \mathrm{h}$. (ver tabla para ubicar los MET)

Kcal. $/ \mathrm{min} .=8$ x 0,0175 x 60 (Kg.) = 8,4 Kcal. $/ \mathrm{min}$.

\section{Diagnóstico del exceso de peso corporal:} sobrepeso y obesidad

Se recomienda utilizar el índice de masa corporal (IMC) para el diagnóstico de exceso de peso corporal sobrepeso u obesidad) en las personas adultas (de edad igual o superior a 18 años) de ambos sexos. El IMC se calcula mediante la fórmula siguiente: peso (en kilogramos)/talla (en metros)2.

El diagnóstico de sobrepeso se establece cuando el IMC es de 25-29,9 y el de obesidad cuando el IMC es mayor o igual a 30. Los criterios de la Organización Mundial de la Salud para la clasificación del peso corporal en adultos según el IMC son los siguientes (tabla 3):

Tabla 3. Clasificación del tipo de obesidad según el índice de masa corporal (IMC).

\begin{tabular}{|c|c|l|}
\hline & $\begin{array}{c}\text { Tipo de } \\
\text { obesidad }\end{array}$ & \multicolumn{1}{|c|}{ IMC (Kgr/m2) } \\
\hline Bajo peso & & Menos de 18,5 \\
\hline Normal & & Entre 18,5 y 24,9 \\
\hline Sobrepeso & I & Entre 25 y 29,9 \\
\hline $\begin{array}{c}\text { Obesidad } \\
\text { Obesidad } \\
\text { extrema }\end{array}$ & II & Entre 30 y 34,9 \\
\hline
\end{tabular}

Las personas adultas con IMC de 18,5-34,9 que tienen una circunferencia de la cintura (CC) mayor de $88 \mathrm{~cm}$ en la mujer y mayor de $102 \mathrm{~cm}$ en el varón son más propensas a padecer hipertensión arterial, diabetes mellitus tipo 2, dislipemia y síndrome metabólico (Arrizabalaga et al., 2004).

- En personas adultas con un IMC entre 25 y 34,9 , la existencia de una CC superior a $88 \mathrm{~cm}$ en la mujer y a $102 \mathrm{~cm}$ en el varón indica un alto riesgo relativo de desarrollar enfermedades asociadas al exceso de peso corporal y a la acumulación excesiva de grasa abdominal.

- Cuando el IMC es igual o mayor de 35, las personas obesas suelen superar los citados puntos de corte de la CC y esta pierde su poder predictivo creciente de riesgo de desarrollo de enfermedades asociadas a la acumulación excesiva de grasa abdominal.

- Cuando una persona adulta presenta un IMC igual o mayor de 25 o tiene una CC mayor de 88 $\mathrm{cm}$ si es mujer o de $102 \mathrm{~cm}$ si es varón, se debe proceder a la detección de factores de riesgo o enfermedades asociadas, valorando en este caso su repercusión orgánica, para determinar la situación de riesgo absoluto mediante la suma de los factores de riesgo.

\section{ENVEJECIMIENTO Y SALUD}

El envejecimiento humano es un fenómeno universal e inevitable. La esperanza media de vida ha mejorado en el último siglo. Actualmente, alrededor del 7\% de la población mundial es de 65 años y más de edad. En los países desarrollados, este porcentaje es aún mayor (15\%) y continúa creciendo (Landínez, Contreras y Castro, 2012).

El envejecimiento en los seres humanos se asocia con una pérdida de la función neuromuscular y del rendimiento, en parte relacionadas con la reducción de la fuerza y la potencia muscular (Macaluso y De Vito, 2004) causada por una pérdida de la masa de los músculos esqueléticos (sarcopenia) (Hunter, McCarthy y Bamman, 2004) y los cambios en la arquitectura muscular. Esta disminución en la fuerza y la potencia muscular, junto con otros factores como el envejecimiento del sistema nervioso somatosensorial y motor (Shaffer y Harrison, 2007), tiene implicaciones funcionales, como disminución en la velocidad al caminar, aumento del riesgo de caídas y una reducción de la capacidad para llevar a cabo las actividades de la vida diaria (AVD). Todo esto contribuye a una pérdida de la independencia y a una reducción en la calidad de vida de las personas (Doherty, 2003).

Multitud de trabajos han estudiado los efectos de la actividad física en los adultos, bien como reducción de la mortalidad (Chakravarty, Hubert, Lingala y Fies, 
2008), como mejora funcional, física y/o psicosocial (Ruiz y Baena, 2011; Walsh, 2011), e incluso como mejora de la calidad de vida (Motl y McAuley, 2009). A pesar de esto, en la investigación llevada a cabo por Cavill, Kahlmeier y Racioppi (2006), los autores afirman que dos tercios de la población adulta no alcanzan el nivel recomendado de actividad física de una hora de intensidad moderada durante, al menos, cinco días a la semana.

La inactividad física es un importante factor de riesgo para muchas condiciones y problemas crónicos de salud, como las enfermedades cardiovasculares, la hipertensión, la obesidad, la osteoporosis, la diabetes mellitus y de salud mental (Christmas y Andersen, 2000).

\section{MODELOS DE SALUD, BIENESTAR Y CALIDAD DE VIDA}

Son muchas las personas en el mundo actual que dedican su esfuerzo y estudio a lo que podría denominarse como la nueva cultura de la longevidad. En realidad, es el intento de vivir más y en mejores condiciones físicas, sociales y mentales, producto de que el avance social está orientado hacia esa dirección, buscando así un modelo de envejecimiento competente en un sentido útil y productivo, capaz de fortalecer desde un punto de vista genérico de la salud su calidad de vida (Ríos et al., 2000). Se define la calidad de vida como un estado de bienestar; sin embargo, esta noción tomará diferentes énfasis según el contexto del cual parte su valoración (Faden y Germán, 1994). Giusti (1991) define la calidad de vida como un estado de bienestar físico, social, emocional, espiritual, intelectual y ocupacional que le permite al individuo satisfacer apropiadamente sus necesidades individuales y colectivas. Además de estas consideraciones, se toma como una variable importante el nivel de actividad física de los sujetos.

La Organización Mundial de la Salud (OMS, 1948) define la salud como: "un estado de completo bienestar físico, psíquico y social y no meramente la ausencia de enfermedad". Por otro lado, la literatura sobre la relación entre la actividad física y la salud y el bienestar apunta claramente a que esta relación es consistente, hasta el punto de considerar el sedentarismo como un factor de riesgo importante para la salud (Arruza et al., 2008).

Frente a la definición propuesta por el modelo médico, que concibe implícitamente la ausencia de enfermedad como una condición necesaria y suficiente para la pre- sencia de salud, la OMS (1948) propone que la salud es un estado de completo bienestar no solo consistente en la mera ausencia de enfermedad, es a partir de este concepto en que basan y surgen diversos modelos.

Como podemos deducir de las definiciones anteriormente nombradas, las variables que involucran la perspectiva subjetiva de la calidad de vida se entenderán de la siguiente forma:

- Autonomía estará definida como capacidad funcional, que se refiere a si puede o no realizar actividades básicas e instrumentales.

- El soporte social, que se refiere a las estrategias (prestar ayuda, colaboración, relaciones familiares) que permiten relacionarse con los demás.

- Salud mental, que involucra el grado de armonía psicoemocional.

- La actividad física como la satisfacción y la disponibilidad para la realización de dichas actividades.

Se han planteado diversos modelos que han puesto de relieve diversos aspectos significativos que pretenden explicar la concepción subjetiva de la salud y de forma análoga del bienestar psicológico:

Modelo del estado completo de salud (Keyes, 2005)

Este da un paso más en la separación conceptual de la salud y la enfermedad; propone que la enfermedad y la salud mental son dos dimensiones unipolares diferentes aunque relacionadas. Este axioma, que choca con la concepción actual existente de facto en la mayor parte de disciplinas científicas sobre la relación salud-enfermedad, tiene importantes consecuencias: si la salud y la enfermedad se consideran dimensiones diferentes, la ausencia de enfermedad no debería ser considerada como una condición necesaria para la presencia de salud definida como un estado de bienestar físico, mental y social. La definición de salud propuesta por la OMS indica que esta no solo se trata de un estado caracterizado por la ausencia de enfermedad, sino por la presencia de algo positivo. Para concretar en qué consiste este estado positivo, Keyes (2005) ha definido la salud mental como un "conjunto de síntomas de hedonía y funcionamiento positivo operativizado por medidas de bienestar subjetivo (percepciones y evaluaciones que las personas hacen sobre su vida y sobre la calidad de su funcionamiento)". 
El modelo de Keyes está compuesto por un total de trece síntomas (medidas) de salud mental. Los análisis factoriales realizados sobre estas medidas replican las estructuras previamente propuestas sobre el bienestar, que señalan la existencia de dos factores: bienestar hedónico (hedonía) y bienestar eu-daimónico (funcionamiento positivo). El modelo se sostiene sobre dos novedosos axiomas centrales:

- Defender que la salud y la enfermedad son dos dimensiones unipolares correlacionadas entre sí más que dos polos de una única dimensión bipolar. Por tanto, la ausencia del trastorno no garantiza la presencia de salud, y viceversa.

- La presencia de salud mental supone la existencia de un funcionamiento psicosocial positivo (e .g. metas claras en la vida, alta resiliencia, relaciones positivas e íntimas con otras personas, sentimiento de pertenencia, confianza en la sociedad y en sus instituciones, etc.).

Para comprobar empíricamente estos dos axiomas, Keyes (2005) llevó a cabo un interesante estudio en el que demostró mediante un análisis factorial confirmatorio que las medidas de salud mental (bienestar subjetivo, bienestar psicológico y bienestar social) y las medidas de enfermedad mental (episodio depresivo mayor, ansiedad generalizada, trastorno de pánico y dependencia del alcohol) constituyen dos dimensiones unipolares diferentes, aunque correlacionadas. Además, las personas completamente saludables mostraron metas más claras, una mayor resiliencia, mayor intimidad en sus relaciones sociales y faltaron menos al trabajo, lo cual supone la principal evidencia empírica para la defensa del segundo axioma.

Tabla 4. Modelo del estado completo de salud (Keyes, 2005).

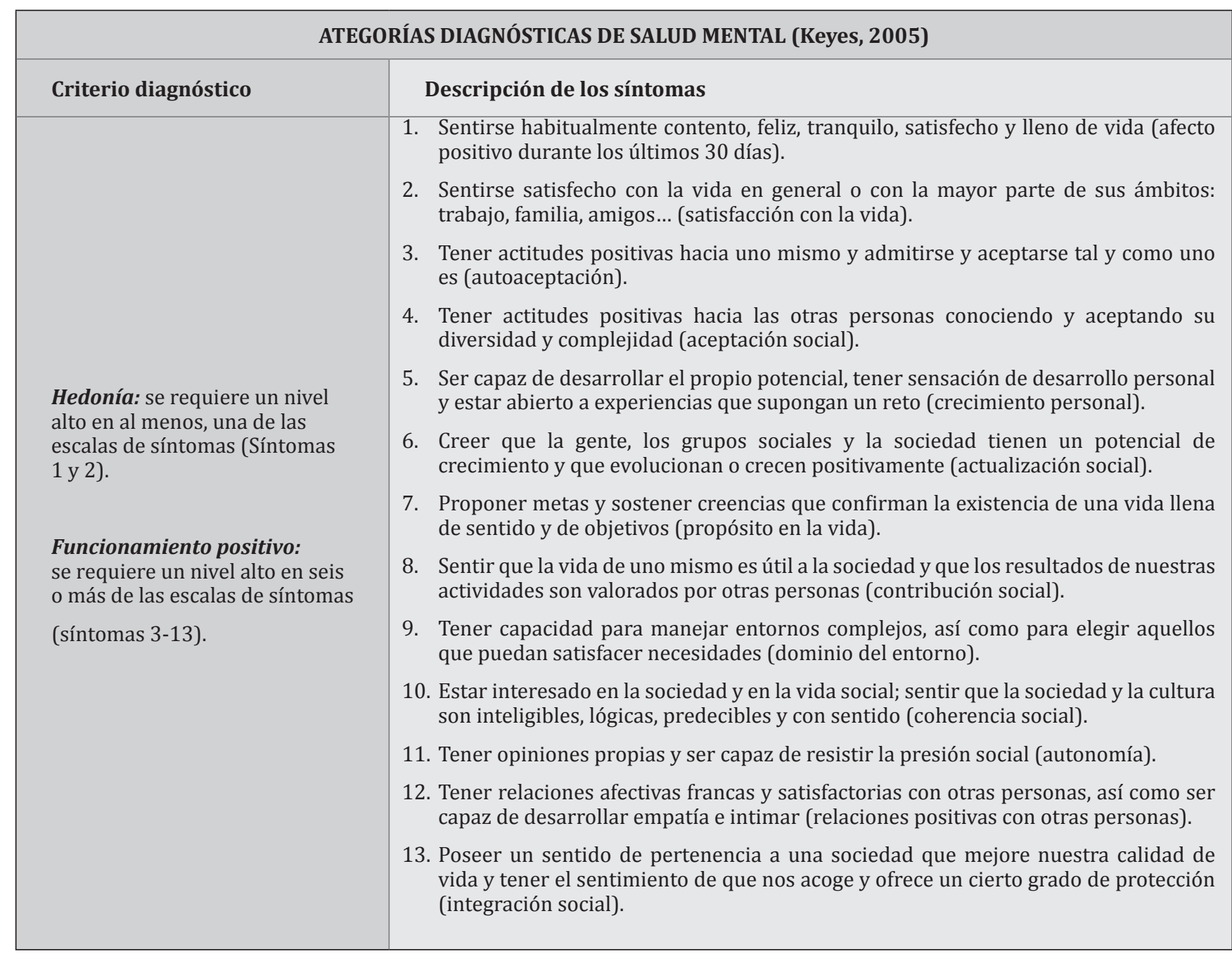




\section{Modelo de creencias en salud de Hochbaum (1958)}

Este modelo fue desarrollado con la intención de explicar por qué las personas no participan en programas preventivos de salud pública. Posteriormente, se adaptó para tratar de explicar una variedad de conductas relacionadas con la salud, entre otras, la respuesta individual ante ciertos síntomas de enfermedad (Weinstein, 1988), el cumplimiento de pacientes con los tratamientos y las recomendaciones médicas (Kirscht, 1974), la práctica de autoexámenes exploratorios (Becker, 1974), el uso del tabaco (Calnan y Moss, 1984) y la participación de inmigrantes latinos en los Estados Unidos en programas de prevención de la tuberculosis. Los factores del modelo que determinan la conducta de salud son de dos tipos: la percepción de amenazas sobre la propia salud y las creencias de los individuos sobre la posibilidad de reducir esas amenazas.

La percepción de amenazas sobre la propia salud se encuentra determinada por los valores generales sobre la salud, las creencias específicas sobre la propia vulnerabilidad ante la enfermedad (percepción de vulnerabilidad) y las creencias sobre la gravedad de la enfermedad (percepción de severidad); mientras que las creencias sobre la posibilidad de reducir la amenaza se encuentran en función de la creencia en la eficacia de las medidas concretas para reducir las amenazas y la convicción de que los beneficios de la medida superan los costos (percepción de beneficios y barreras). Una última variable que completa el modelo es la presencia de estímulos internos o externos que se constituyen en claves para actuar. Una clave interna puede ser el síntoma de una enfermedad, mientras una clave externa puede ser una campaña acerca de la promoción en salud o las interacciones sociales con amigos afectados por alguna enfermedad. Adicionalmente, el concepto de autoeficacia ha sido adherido a algunas versiones del modelo de creencias de salud. La autoeficacia es útil para entender comportamientos relacionados con el cuidado de la enfermedad crónica que requiere cambios comportamentales en un largo periodo (Beneit, 1994). De acuerdo con este modelo, las creencias sobre la importancia o gravedad de un determinado problema, la vulnerabilidad frente a ese problema y que la acción a implementar produzca más beneficios que costos personales favorecen la conservación y mejoramiento de la salud, la evitación de conductas de riesgo, la prevención de las enfermedades y lo que en general puede denominarse la adopción de estilos de vida saludables.
Teoría de la acción razonada (Fishbein y Ajzen, 1975)

Esta teoría intenta integrar factores actitudinales y comportamentales para explicar conductas de salud. El planteamiento del modelo es que una conducta de salud es resultado directo de la intención comportamental, que depende, a su vez, de las actitudes que se tengan respecto al comportamiento concreto y de las valoraciones o normas subjetivas con respecto a lo apropiado de la acción comportamental. El supuesto del modelo es que los seres humanos somos seres racionales y aplicamos información disponible de manera sistemática para evaluar el costo beneficio de una acción particular. El objetivo de la teoría es ser capaz de predecir y entender directamente los comportamientos observables que están primariamente bajo el control de los individuos.

Modelo de adopción de precauciones (Weinstein, 1988)

Este modelo plantea la existencia de cinco etapas que buscan identificar, valorar y fomentar los factores de protección para la salud:

- La percepción del riesgo, de la severidad y la susceptibilidad, así como el valor de las precauciones.

- El reconocimiento de estos factores para otras personas como pares o grupos de referencia.

- La percepción de los factores en sí mismo o aceptación de la susceptibilidad personal.

- La decisión de adoptar la precaución.

- La toma de la precaución.

Ryff (1989a) plantea que las diversas perspectivas que existen respecto al bienestar subjetivo pueden ser integradas, en la medida que muchos teóricos coinciden en ciertas características de este; Ryff (1989b) racionaliza el bienestar subjetivo en las siguientes dimensiones:

- Autoaceptación es el criterio más utilizado para definir bienestar y es conceptualizado como la figura central de la salud mental, como una característica de madurez, realización personal y funcionamiento óptimo.

- Relaciones positivas con los demás, la importancia de la calidez y confianza en las relaciones interpersonales, así como la capacidad de amar, son vistas como uno de los principales componentes de la salud mental, siendo a la vez un criterio de madurez. 
- Autonomía, enfatiza la autodeterminación, la independencia y la regulación de la conducta.

- Dominio del ambiente, la habilidad de elegir o crear ambientes acordes con las propias condiciones físicas es definida como una característica de la salud mental. Implica la capacidad de manipular y controlar los entornos o ambientes complejos contando con la participación del medio.

- Propósito en la vida, destaca la comprensión de un propósito o significado de la vida, un sentido de dirección o intencionalidad. Quien funciona positivamente tiene objetivos, intenciones y un sentido de dirección.

- Crecimiento personal, el funcionamiento psicológico óptimo requiere no solo desarrollar las características anteriores, sino también continuar el desarrollo de su propio potencial, crecer y expandirse como persona.

Los procesos de envejecimiento y el cúmulo de pérdidas psicosociales que acontecen durante la vejez parecen determinar en algunas personas mayores su incapacidad para percibir sus competencias y habilidades $\mathrm{y}$, desde luego, los aspectos positivos del entorno que les rodea y de la vida en general. En este sentido, existen hallazgos empíricos que demuestran que la autoestima decrece con la edad. Esta autoestima o autosatisfacción puede considerarse como el eje de la calidad de vida de los adultos/ancianos. Mroczek y Kolarz (1998) señalan la importancia de algunas variables sociodemográficas contextuales y de personalidad en la relación edad-bienestar. En particular, estos autores encuentran que se presentan mejores índices de bienestar en los adultos mayores casados y extrovertidos, en detrimento de los solteros e introvertidos, no encontrándose relaciones lineales en el caso de las mujeres. Sin embargo, la mayoría de los adultos mayores permanecen socialmente integrados, son competentes y las posibilidades de que presenten deficiencias en algunos aspectos pueden ser modificables. Es evidente que existen múltiples diferencias individuales en el bienestar percibido en la vejez y en definitiva no es exactamente cierto que a medida que envejecen las personas se sientan más insatisfechas; es decir, la edad cronológica no parece explicar sino una pequeña porción de la varianza en el grado de bienestar del sujeto (Stanley y Cheek, 2003).
Por otra parte, integrando la descripción hecha por Ryff (1989a) en torno al concepto de bienestar, nos podemos dar cuenta de que al menos la autoaceptación, la autoestima y el propósito en la vida tienen que ver con muchos de los síntomas asociados comúnmente a la depresión, así como el dominio del ambiente y las relaciones positivas con los demás se relacionan con el concepto de apoyo social percibido. Ryff y Keyes (1995) destacan la importancia de la relación entre las expectativas de los individuos y los logros en la obtención del bienestar.

\section{EJERCICIO FÍSICO Y SENTIMIENTO DE BIENESTAR EN LAS PERSONAS ADULTAS}

Los gerontólogos dividen a los adultos mayores en esta etapa en dos bloques: los viejos-jóvenes (entre 65 y 74 años) y los viejos-viejos (a partir de los 75). Según Villar, Triado, Solé y Osuna (2003), las personas mayores se encuentran situadas en un momento vital en el que la vida está limitada en un sentido cuantitativo, ya que las probabilidades de enfermedad y disfunción aumentan, por lo cual se torna dificultoso el estudio de la calidad de vida en dicho grupo de población; sin embargo, para Yanguas (2006), la calidad de vida es un concepto multidimensional que comprende componentes tanto objetivos como subjetivos, incluyendo un número diverso de ámbitos de la vida, refleja las normas culturales de bienestar objetivo y otorga un peso específico diferente a los distintos ámbitos de la vida considerados más importantes para algunas personas que para otras. En este mismo sentido, Terol et al. (2000) indican que existe acuerdo en cuanto a que la calidad de vida, como un todo, debe evaluarse atendiendo a aspectos físicos, sociales y psicológicos, por un lado, y, por otro lado, a la subjetividad de quien informa, es decir, como el sujeto los percibe.

La actividad física está siendo cada vez más estudiada en nuestra sociedad debido, entre otros aspectos, a los beneficios que produce en la salud y en la calidad de vida de las personas (Motl y McAuley, 2009). En contraposición, la inactividad se está abordando desde diversos paradigmas debido a que se ha determinado como un alto factor de riesgo, capaz de producir gran cantidad de enfermedades (Lobelo, Pate, Parra, Duperly y Pratt, 2006). Desde el paradigma orientado a la actividad física (Devís y Peiró, 1993), se entiende 
la actividad física como un elemento integrador junto con la herencia genética, el estilo de vida, el ambiente que rodea a los individuos y otros atributos personales como la familia, que, obviamente, pueden determinar la salud de las personas.

El ejercicio físico provoca efectos beneficiosos desde el punto de vista fisiológico, psicológico y social. Una parte de los beneficios que se le atribuyen se basan en las rigurosas mediciones antropométricas, bioquímicas y fisiológicas realizadas a los sujetos, mientras que otros resultan de reportes de auto-observación, en este último caso se incluyen los beneficios subjetivos percibidos por el practicante.

El envejecimiento se acompaña de un conjunto de efectos que disminuyen la aptitud y el rendimiento físico, muchos de los cuales resultan de la disminución de la actividad motriz. A partir de la séptima década de vida, ocurre una declinación acelerada de la capacidad funcional. Sin embargo, el envejecimiento es un proceso fisiológico que no corre necesariamente en forma paralela a la edad cronológica y presenta una considerable variación individual. Un ejemplo de ello es la denominada sarcopenia, es decir, la pérdida de masa muscular asociada con el envejecimiento; esta puede deberse a factores muy diversos, como los cambios estructurales del aparato músculoesquelético, las enfermedades crónicas y sus tratamientos y la atrofia por desuso o la malnutrición (Kamel, 2003). El nivel de fuerza necesario para satisfacer las exigencias de la vida cotidiana no varía demasiado a lo largo de la vida. No obstante, el proceso de envejecimiento y la sarcopenia traen consigo una dis- minución en la producción de fuerza máxima, lo que dificulta la realización de las tareas que antes hacíamos fácilmente. La fuerza muscular es necesaria para poder realizar las más diversas tareas del día a día, como levantarse de la cama, asearse, subir escaleras o simplemente andar y la disminución de esta cualidad física es una de las causas principales de la pérdida del equilibrio en personas mayores, que trae consigo una propensión a las caídas y un riesgo incrementado de fracturas de los huesos osteoporóticos (Roubenoff, 2000).

La disminución progresiva de la masa muscular, así como la debilidad que ocurre en los músculos con el envejecimiento, contribuyen también a la pérdida de dinamismo. Este deterioro es además causal de la merma de independencia en el anciano, de una mayor demanda de servicios sanitarios, aislamiento social, depresión y abandono (Greenlund y Fair, 2003). Como consecuencia de las severas implicancias que este fenómeno de incapacidad genera, se viene prestando una especial atención al entrenamiento de la fuerza como el medio para controlar la pérdida de la musculatura esquelética que ocurre con la edad. El aumento de fuerza y masa muscular obtenidos supone una ganancia de independencia funcional y por tanto una mejora en la calidad de vida, lo que implica menor dependencia de otras personas y, obviamente, reducción del riesgo de contraer patologías musculoesqueléticas y, secundariamente, otras de índole metabólica.

Entre los efectos ocasionados por el proceso de envejecimiento en las personas destacan:

Tabla 5. Efectos ocasionados por el proceso de envejecimiento.

Fuente: adaptado de Carbonell et al. (2009); Barrios et al. (2003); Terreros (1992); Schlüssel et al. (2008).

\begin{tabular}{|l|l|}
\hline Disminución de la estatura. & Disminución de la densidad ósea. \\
\hline Disminución de la masa muscular. & Un mayor índice de fatiga muscular. \\
\hline Pérdida de fuerza. & Disminución del gasto cardiaco. \\
\hline $\begin{array}{l}\text { Notable disminución del número y tamaño de las } \\
\text { fibras musculares. }\end{array}$ & $\begin{array}{l}\text { Disminución del consumo de oxígeno y su } \\
\text { utilización por los tejidos. }\end{array}$ \\
\hline $\begin{array}{l}\text { Notable disminución de la frecuencia y el volumen } \\
\text { sistólico. }\end{array}$ & $\begin{array}{l}\text { Menor capacidad de adaptación y recuperación del } \\
\text { ejercicio. }\end{array}$ \\
\hline Aumento de la presión arterial. & $\begin{array}{l}\text { Aumento de la frecuencia ventilatoria durante el } \\
\text { ejercicio. }\end{array}$ \\
\hline
\end{tabular}


Disminución de la capacidad vital

Menor tiempo de reacción y menor velocidad de movimientos.

Disminución de la coordinación.

Disminución de la movilidad articular.

Incremento de la grasa corporal.
Disminución de la agilidad.

Los trastornos del equilibrio.

Aumento de la rigidez de cartílagos, tendones y ligamentos.

Resultan comunes los dolores de espalda, fracturas de cadera, problemas respiratorios, la hipertensión arterial, las lesiones osteomioarticulares.

Trastornos angiológicos, digestivos y nerviosos.
Por otra parte, Mazorra (2003) mantiene que la incrementación del tono y la masa muscular, por tanto la fuerza; fomenta la mejoría de las condiciones hemodinámicas y de la mecánica ventilatoria y su eficiencia; la disminución de la tensión arterial; la prevención de la arteriosclerosis, la hiperlipidemia y la osteoporosis; la ayuda en el control de la diabetes; así como la mejoría en la flexibilidad, el equilibrio y la movilidad articular. En la misma línea, Sánchez (2002) sostiene que, entre los beneficios percibidos por los adultos mayores, se han encontrado el propio hecho de sentirse bien, abandono o disminución del consumo de medicamentos y el mejoramiento de los síntomas, mejor conciliación del sueño, aumento de seguridad ante caídas, una mayor distracción y la ampliación de relaciones interpersonales. Por tanto, la práctica de actividad física ayuda a prevenir, gestionar y rehabilitar diferentes condiciones musculoesqueléticas, cardiorrespiratorias, metabólicas, neurológicas y mentales, entre otras (Vuori, 2004). Cuando se cumple con la dosis de actividad física recomendada, se pueden reducir los riesgos de varias enfermedades crónicas no transmisibles, como enfermedades cardiovasculares, ataques al corazón, diabetes tipo II, cáncer de colon, cáncer de mama. Además, el incremento de ciertos tipos de actividad física puede beneficiar a la salud a través de efectos positivos sobre la hipertensión y osteoporosis, mejora la composición corporal, dolor lumbar, las condiciones musculoesqueléticas como la osteoartritis (World Health Organization, 2008).

Heredia (2006) considera que el ejercicio físico practicado regularmente en la tercera edad contribuye a:

Tabla 6. Beneficios del ejercicio regular en la tercera edad.

\begin{tabular}{|c|c|}
\hline Mejorar la capacidad para el auto cuidado. & Frenar la atrofia muscular. \\
\hline Favorecer la integración del esquema corporal. & $\begin{array}{l}\text { Inducir cambios positivos en el estilo de vida de los } \\
\text { adultos mayores. }\end{array}$ \\
\hline Propiciar bienestar general. & Incrementar la calidad del sueño. \\
\hline Conservar más ágiles y atentos nuestros sentidos. & Disminuir la ansiedad, el insomnio y la depresión. \\
\hline Facilitar las relaciones intergeneracionales. & $\begin{array}{l}\text { Reforzar la actividad intelectual, gracias a la buena } \\
\text { oxigenación cerebral. }\end{array}$ \\
\hline $\begin{array}{l}\text { Facilita las relaciones familiares, contactos sociales y la } \\
\text { participación social. }\end{array}$ & Contribuir en gran manera al equilibrio psicoafectivo. \\
\hline Favorecer la movilidad articular. & Prevenir caídas. \\
\hline Evitar la descalcificación ósea. & $\begin{array}{l}\text { Incrementar la capacidad aeróbica, la fuerza } \\
\text { muscular y la flexibilidad. }\end{array}$ \\
\hline $\begin{array}{l}\text { Aumentar la eliminación de colesterol, disminuyendo el } \\
\text { riesgo de arterioesclerosis e hipertensión. }\end{array}$ & $\begin{array}{l}\text { Reducir el riesgo de formación de coágulos en los } \\
\text { vasos y, por tanto, de trombosis y embolias. }\end{array}$ \\
\hline
\end{tabular}


Los beneficios que brinda la actividad físico-deportiva a la salud psicológica no son tan evidentes como los de la salud fisiológica, sin embargo, los estudios en esta línea han revelado que el ejercicio físico mejora el estado de ánimo, disminuye la depresión y la ansiedad, eleva el vigor, la autoestima y mejora el enfrentamiento al estrés de la vida cotidiana (Kravitz, 2007). Una recopilación de beneficios de la actividad físico-deportiva a la salud psicológica realizado por Márquez (1995) menciona que el ejercicio ayuda a reducir el estado de ansiedad, depresión, los niveles de neuroticismo, el estrés, produce efectos emocionales beneficiosos en todas las edades y sexos y mejora los procesos cognitivos, aumentando la actividad cerebral. Además, destaca el empleo del ejercicio como complemento adicional en el tratamiento sobre casos severos de depresión.

Por último, y al igual que en la salud física y psicológica, el entorno social de las personas también se puede beneficiar de la práctica de actividades físico-deportivas. El ejercicio ofrece oportunidades de distracción e interacción social (Barrios et al., 2003), ya que desarrolla el sentido de la responsabilidad, el trabajo en equipo y puede llegar a ser muy útil sí se emplea como antídoto en contra de vicios, la delincuencia y la violencia. Además, se ha comprobado que favorece el aprendizaje de los roles del individuo, las reglas sociales, refuerza la autoestima, el autoconcepto y el sentimiento de identidad y solidaridad (Teixeira y Kalinoski, 2003).

En definitiva, la actividad física mejora la salud física y mental, permitiendo a las personas desarrollarse integralmente en sus diferentes entornos sociales, siendo componentes esenciales, independientes, necesarios e íntimamente relacionados con la calidad de vida positiva (Llopis, 2008).

Se considera que los sujetos que poseen conocimientos y creencias positivas respecto a los beneficios del ejercicio tienden a promover la actividad física dentro de su estilo de vida, pero ello no conduce necesariamente a la incorporación regular al ejercicio. Los beneficios percibidos repercuten en la permanencia de los sujetos incorporados, es decir, existe relación entre motivación y el beneficio percibido; se ha observado que los sujetos con menor nivel educativo y cultural perciben en menor medida estos beneficios y los que practicaron deportes en su juventud perciben más (Barrios et al., 2003).
Son numerosas las investigaciones que avalan los beneficios del ejercicio físico en diferentes ámbitos del bienestar psicológico (Jiménez, Martínez, Miró y Sánchez, 2008): mejora la salud subjetiva, el estado de ánimo y la emotividad, reduce la depresión clínica, disminuye los niveles de ansiedad, favorece el afrontamiento del estrés e incrementa la autoestima.

Los beneficios físicos percibidos por los sujetos confirman que el ejercicio en el adulto mayor contribuye a disminuir dolores, calambres y entumecimientos; los problemas osteomioarticulares son padecidos por un elevado porcentaje de sujetos, se conoce que el ejercicio induce analgesia, caracterizada por una disminución de la respuesta a un estímulo doloroso durante y después de una sesión de ejercicio; mejora el sueño, aunque hay un pequeño número de sujetos que lo perciben, resulta interesante el hallazgo, pues estudios en condiciones experimentales abordan con cautela su papel, solo se llega a afirmar que el ejercicio contribuye a la higiene del sueño. Resulta provechoso para el fortalecimiento de las piernas y el cuerpo en general y el aumento de la seguridad ante las caídas, fenómeno frecuente y peligroso en este tipo de población; por tanto, contribuye a mejorar la calidad de vida; mejora el estado de ánimo y ofrece distracción, el adulto mayor es propenso a sufrir depresiones y pérdida de la autoestima; con la práctica de ejercicio se contribuye a la salud mental y se favorece la interacción social del adulto mayor con su comunidad (Shephard, 1993; Barrios et al. 2003).

Además, entre los beneficios percibidos por la práctica de ejercicio físico por adultos mayores se han encontrado (Sánchez, 2002): el propio hecho de sentirse bien; abandono o disminución del consumo de medicamentos y el mejoramiento de los síntomas; mejor conciliación del sueño; aumento de seguridad ante caídas; una mayor distracción y la ampliación de relaciones interpersonales.

La práctica de la actividad física y deportiva no es un producto del ejercicio en sí mismo, sino que viene motivado además por el seguimiento de hábitos saludables por parte de las personas que lo practican, como una alimentación equilibrada o la disminución de conductas de riesgo, como el consumo de alcohol y tabaco (Jiménez et al. 2008). Las personas que mantienen un estilo de vida físicamente activo o una buena 
forma física tienen menores tasas de mortalidad que sus homónimos sedentarios y una mayor longevidad. Las personas que en cualquier momento de su vida abandonan su hábito sedentario para pasar a otro más activo físicamente, o aumentan su forma física, reducen sus tasas de mortalidad significativamente (Cabezas et al., 2007).

\section{RECOMENDACIONES SOBRE LA ACTIVIDAD FÍSICA PARA MEJORAR LA SALUD EN LAS PERSONAS MAYORES}

Siguiendo las recomendaciones de la OMS (2010), estas directrices son válidas para todos los adultos sanos de 65 años en adelante. Son también aplicables a las personas de ese grupo de edades con ENT crónicas. Las personas con dolencias específicas (por ejemplo, enfermedad cardiovascular o diabetes) podrían tener que adoptar precauciones adicionales y solicitar asesoramiento médico antes de tratar de alcanzar el nivel de actividad física recomendado para los adultos de mayor edad. Estas recomendaciones son aplicables a todos los adultos de mayor edad, con independencia de su género, raza, etnicidad o nivel de ingresos. Para conseguir una eficacia máxima, sin embargo, las estrategias de comunicación, o las formas de difusión y explicación de las recomendaciones, podrían diferir según el grupo de población. También son válidas para los adultos de mayor edad con discapacidades, aunque podría ser necesario adaptarlas a cada individuo en función de su capacidad para hacer ejercicios y de los riesgos o limitaciones específicas para su salud.

1. Los adultos de mayor edad deberían acumular un mínimo de 150 minutos semanales de actividad física aeróbica moderada, o bien no menos de 75 minutos semanales de actividad aeróbica vigorosa, o bien una combinación equivalente de actividad física moderada y vigorosa.

2. La actividad aeróbica se desarrollará en sesiones de diez minutos como mínimo.

3. Para obtener aún mayores beneficios, los adultos de este grupo de edades deberían aumentar hasta 300 minutos semanales su actividad física mediante ejercicios aeróbicos de inten- sidad moderada, o bien practicar 150 minutos semanales de actividad aeróbica vigorosa, o bien una combinación equivalente de actividad física moderada y vigorosa.

4. Los adultos de mayor edad con dificultades de movilidad deberían dedicar tres o más días a la semana a realizar actividades físicas para mejorar su equilibrio y evitar las caídas.

5. Deberían realizarse actividades de fortalecimiento muscular de los grandes grupos musculares dos o más veces a la semana.

6. Cuando los adultos de este grupo no puedan realizar la actividad física recomendada debido a su estado de salud, deberían mantenerse activos hasta donde les sea posible y les permita su salud.

Si nos basamos en los diferentes parámetros (frecuencia, duración, intensidad, tipo de ejercicio) para obtener un beneficio sobre los diferentes niveles de salud, el ACSM (2001) recomienda que se practique ejercicio físico con:

- Una frecuencia entre 3-5 días por semana para conseguir mejoras significativas en el estado de salud.

- En cuanto a la duración, parece haber cierta coincidencia en el intervalo 20-60 minutos por sesión, o bien lo suficiente para provocar un gasto energético entre 200 y 300 kilocalorías (Miguel, Martín y Navlet, 2001).

- En cuanto a la intensidad, debería adecuarse a la forma física de cada persona. En todo caso, un ejercicio saludable sería aquel que se encontrara entre el 50\% y el 85\% del consumo máximo de oxígeno (Van Amersfoort, 1996).

- Debemos tener claro que la cantidad, más que la intensidad de la actividad físico-deportiva, influye sobre los estados de salud. De hecho, parece que no es necesario realizar una actividad física vigorosa para mejorar sus efectos sobre la salud, aunque deben complementarse en las sesiones ejercicios de resistencia/aeróbicos, de fuerza, flexibilidad y equilibrio. 
La evidencia obtenida en función de los marcadores biomédicos indica que sería beneficioso realizar actividades físicas con regularidad a lo largo de la semana (por ejemplo, cinco o más veces semanalmente). Con ello se favorecería también la integración de la actividad física en la vida cotidiana, por ejemplo mediante paseos a pie o en bicicleta.

La cantidad y calidad de ejercicio necesarias para alcanzar beneficios relacionados con la salud pueden diferir de lo que se recomienda para los beneficios de la aptitud física, de forma que los niveles bajos de actividad física pueden reducir el riesgo de ciertas enfermedades crónico degenerativas y mejorar la condición metabólica y aún así no ser de suficiente cantidad o calidad para mejorar el máximo consumo de oxígeno.

\section{CONCLUSIONES Y DISCUSIÓN}

A la luz del concepto de salud actual, la actividad física no puede ser considerada únicamente como un remedio para curar o prevenir enfermedades diversas, sino que también debe concebirse en relación con una sensación subjetiva de salud que redunde en el bienestar (Devís, 2000). La salud se vincula, de este modo, al concepto de calidad de vida y a la percepción que los individuos tienen de su estado de bienestar físico y psicosocial. Ciertamente, es preocupante que cada año que transcurre tengamos más personas mayores con mala salud (y peor bienestar); a esta situación no se ha llegado por un solo motivo, pero creemos que uno muy importante ha sido la disminución de la calidad en la relación equipo de salud (médico-usuario). Entendemos que el excesivo énfasis en los aspectos tecnológicos y/o farmacológicos y sobre todo el deterioro de la comunicación profesionales/usuario, en parte por la escasez de tiempo y también debido la escasa formación de los profesionales en este ámbito, ha ido restándole importancia a la relación de ayuda/consejo profesional, que fue, en otro momento, el soporte social para el usuario y fuente de gratificación y reconocimiento para el profesional de la salud. El diagnóstico y el tratamiento de la enfermedad, a nivel biomédico exclusivamente, el uso de complicados procedimientos tecnológicos y el descanso en la farmacología casi exclusivamente, que sin duda han representado un salto cualitativo a nivel de la supervivencia en enfermedades antes rápidamente mortales, han dejado de lado, en muchas ocasiones, la aproximación más holística al cuidado de la salud, donde no solo se busque combatir la enfermedad (y en muchas ocasiones retrasar la muerte), sino promover el bienestar (Schwartzmann, 2003). De hecho, medidas como el consejo médico a la población en general, y a los mayores en particular, para la realización de actividad física supone una vía económica, eficaz y válida para aumentar el bienestar de la población sin excepciones. Por tanto, creemos firmemente que se debe fomentar en la población adulta el ejercicio físico, ya que las implicaciones positivas que acarrea son, en ocasiones, muy superiores a las farmacológicas.

\section{REFERENCIAS BIBLIOGRÁFICAS}

Abrante, C., Brito, O. y García, C. (1996). El deporte en edad avanzada. Programa de actividades físicas y deportivas para personas mayores. Gran Canaria: Editorial Nogal.

American College of Sport Medicine (ACSM). (2001). Position Stand on Exercise and Physical Activity for Older Adults. Medicine and Sciences in Sports and Exercise, 30, 992-1008.

Arrizabalaga, J. et al. (2004). Recomendaciones y algoritmo de tratamiento del sobrepeso y la obesidad en personas adultas. Med Clin (Barc), 122(3), 104-110.

Arruza, J., Arribas, S., Gil de Montes, L., Irazusta, S., Romero, S. y Cecchini, J. (2008). Repercusiones de la duración de la actividad físico-deportiva sobre el bienestar psicológico. Revista Internacional de Medicina y Ciencias de la Actividad Física y el Deporte, 8(30), 171-183.

Barrios, R., Borges, R. y Cardoso, l. (2003). Beneficios percibidos por adultos mayores incorporados al ejercicio. Revista Cubana Medicina General Integral, 19(2), 24-34.

Becker, M. (1974). The health belief model and personal health behavior. Health Educ Monogr, 2, 10-24.

Beneit, P. (1994). Conductas de salud. En: J. Latorre y P. Beneit, Psicología de la salud. Buenos Aires: Editorial Lumen.

Cabezas, C., Robledo, T., Marquesa, R., Ortega, M., NebotAdella, M., Megido, E., Muñoz, J., Prados, J.y Córdoba, R. (2007). Grupos de Trabajo de Educación Para la Salud. Atención Primaria, 39(3), 27-46.

Calnan, M. y Moss, S. (1984).The health belief model and compliance with education given at a class on breast self examination. J Health Soc Behavior, 25, 198-210.

Carbonell, A., Aparicio, V. y García-Molina, M. (2009). Efectos del envejecimiento en las capacidades físicas: implicaciones en las recomendaciones de ejercicio 
físico en personas mayores. International Journal of Sport Science, 4(17), 1-18.

Cavill, N., Kahlmeier, S. y Racioppi, F. (2006). Physical activity and health in Europe: evidence for action. World Health Organization: Copenhagen.

Chakravarty, E., Hubert, H., Lingala, V. y Fries, J. (2008). Reduced disability and mortality among aging runners. Archives of Internal Medicine, 168, 1638-1646.

Christmas, C. y Andersen, R.A. (2000). Exercise and older patients: guidelines for the clinician. J Amer Geriatrics Soc, 48, 318-324.

Devís, J. (2000). Actividad física, deporte y salud. Barcelona: INDE.

Devís, J. y Peiró, C. (1993). La actividad física y la promoción dela salud en niños/as y jóvenes: la escuelay la Educación Física. Revista de Psicología del Deporte, 4, 71-86.

Díaz, P., Puente, G. y García, T. (1994). Resultados de la aplicación de una escala de valoración sociofamiliar en atención primaria. Revista Española de Geriatría y Gerontología, 29, 239-245.

Doherty, T. (2003). Invited review: aging and sarcopenia. J App Physiol, 95(4), 1717-1727.

Faden, R. y Germán, P. (1994). Calidad de vida. Consideraciones en geriatría. Clínica de Medicina Geriátrica. México: Editorial Interamericana.

Fishbein, M. y Ajzen, I. (1975). Belief, attitude, intention and behavior: an introduction to theory and research. Reading: Addison-Wesley Publishing.

García Ferrando, M. (2006). Posmodernidad y deporte: entre la individualización y la masificación. Encuesta sobre hábitos deportivos de los españoles 2005. Madrid: CSD y CIS.

Giusti, L. (1991). Calidad de vida, estrés y bienestar. San Juan, Puerto Rico: Editorial UCR.

Greenlund, L. y Fair, K. (2003). Sarcopenia: consequences, mechanisms and potential therapies. Mechanisms of Ageing and Development, 124, 287-299.

Heredia, L. (2006). Ejercicio físico y deporte en los adultos mayores. Geroinfo: publicación de gerontología y geriatría. Recuperado de http://bvs.sld.cu/revistas/ geroinfo/vol1_4_06/geroinfo2110.pdf

Hochbaum, G. (1958). Public participation in medical screening programs: A sociopsychological study. Washington, D.C.: US Government Printing Office, PHS, 572.

Hunter, G., McCarthy, J.y Bamman, M. (2004). Effects of resistance training on older adults. Sports Med, 34(5), 329-348.

Jackson, A., Morrow, J., Hill, D. y Dishman, R. (2003). Physical activity for health and fitness. Human Kinetics: Champaign.
Jiménez, M., Martínez, P., Miró, E. y Sánchez, A. (2008). Bienestar psicológico y hábitos saludables: ¿están asociados a la práctica de ejercicio físico? International Journal of Clinical and Health Psychology, 8(1), 185-202.

Jiménez, Y., Núñez, M. y Coto, E. (2013). La actividad física para el adulto mayor en el medio natural. Intersede, 19(27), 168-181.

Keyes, C. (2005). Mental Illness and/or Mental Health. Investigating Axioms of the Complete State Model of Health. Journal of Consulting and Clinical Psychology, $73,539-548$.

Kirscht, J. (1974). The health belief model and illness behavior. Health Educ Monogr, 2, 387- 408.

Kravitz, L. (2007). The 25 Most Significant Health Benefits of Physical Activity \& Exercise. IDEA Fitness Journal, $4(9)$, 54-63.

Landínez, N., Contreras, K. y Castro, A. (2012). Proceso de envejecimiento, ejercicio y fisioterapia. Revista Cubana de Salud Pública, 38(4), 562-580.

Leturia, F. (1998). Jubilación y calidad de vida. Geriátrica Gerontológico, 33, 9-16.

Llopis, D. (2008). Salud mental y ejercicio físico. En Arufe, Domínguez, García y Lera (ed.), Ejercicio físico, salud y calidad de vida (pp. 178-198). Sevilla: Wanceulen.

Lobelo, F., Pate, R., Parra, D., Duperly, J. y Pratt, M. (2006). Carga de mortalidad asociada a la inactividad física en Bogotá. Revista Salud Pública, 8(2), 28-41.

Macaluso, A. y De Vito, G. (2004). Muscle strength, power and adaptations to resistance training in older people. Eur J App Physiol, 91(4), 450-472.

Márquez, S. (1995). Beneficios psicológicos de la actividad física. Revista de Psicología General y Aplicada, 48, 185-206.

Márquez, S., Rodríguez, J. y De Abajo, S. (2006). Sedentarismo y salud: efectos beneficiosos de la actividad física. Apuntes Educación Física y Deportes, 83(19), 12-24.

Mazorra, R. (2003). Actividad física y salud. La Habana: Científico-Técnica.

Miguel, F., Martín, M. y Navlet, M. (2001). El efecto del ejercicio físico sobre la ansiedad y la depresión. Revista Española e Iberoamericana de Medicina de la Educación Física y el Deporte, 10, 49-57.

Ministerio de Sanidad y Política Social. (2010). Indicadores de Salud 2009. Evolución de los indicadores del estado de salud en España. Madrid.

Moreno, J., Martínez-Galindo, C., González-Cutre, D. y Cervelló, E. (2008). Motivación hacia la práctica físicodeportiva en personas mayores. En: E. Martín y R. Gomes de Sousa (ed.), Actividade física e envelhecimento saudável (pp.153-169). Río de Janeiro: Shape. 
Motl, R. y McAuley, E. (2009). Pathways between physical activity and quality of life in adults with multiple sclerosis. Health Psychology, 28(6), 682-689.

Mroczek, D. y Kolarz, C. (1998). The effect of age on positive and negative affect: A developmental perspective on happiness. Journal of Personality and Social Psychology, $75,1333-1349$.

Organización Mundial de la Salud OMS (2010). Recomendaciones mundiales sobre actividad física para la salud. OMS: Ginebra.

Organización Mundial de la Salud. OMS (1948). World Health Organization Constitution. En Basic Documents. Ginebra. Suiza.

Physical activity and patient-reported outcomes: enhancing impact. (2013). European Review of Aging and Physical Activity, 10(1), 37+.

Ríos, L., Ríos, I. y Padial, P. (2000) La actividad física en la tercera edad. Recuperado de http://www.efdeportes. com/efd18/3aedad1.htm)

Roubenoff, R. (2000). Sarcopenia and its implications for the elderly. European Journal of Clinical Nutrition, 54, 40-47.

Ruiz, F. y Baena, A. (2011). Efectos del ejercicio aeróbico sobre los estados de ánimo en mujeres mayores. Retos. Nuevas tendencias en Educación Física, Deporte y Recreación, 20, 43-47.

Ruiz, F., Ruiz-Risueño, J., De la Cruz, E. y García, M. (2009). Actividad física y consumo de alcohol en adultos. Retos. Nuevas tendencias en Educación Física, Deporte y Recreación, 16, 46-50.

Ryff, C. (1989a). Beyond Ponce de Leon and life satisfaction: New directions in quest of successful aging. International Journal of Behavioral Development, $12,35-55$.

Ryff, C. (1989b). Happiness is everything, or is it. Explorations on the meaning of psychological wellbeing. Journal of Personality and Social Psychology, 57, 1069-1081.

Ryff, C. y Keyes, C. (1995). The structure of psychological well-being revisited. Journal of Personality and Social Psychology, 69, 719-727.

Sánchez, P. (2002). Beneficios percibidos y adherencia a un programa de actividad física gerontológica. Lecturas: Educación Física y Deportes, 8(52). Recuperado de http//www.efdeportes.com

Kamel, H. (2003). Sarcopenia and aging. Nutrition Reviews, 61, 157-167.

Schlüssel, M., Dos Anjos, L., De Vasconcellos, M. y Kac, G. (2008). Reference values of handgrip dynamometry of healthy adults: a population-based study. Clin Nutr; 27, 601-607.

Schwartzmann, 1. (2003). Calidad de vida relacionada con la salud: aspectos conceptuales. Ciencia y Enfermería, 9(2), 9-21.
Shaffer, S.y Harrison, A. (2007). Aging of the somatosensory system: a translational perspective. Physical Ther, 87(2), 193-207.

Shephard, R. (1993). Exercise and aging: Extending independence in older adults. Geriatric, 5, 61-64.

Stanley, M. y Cheek, J. (2003). Well-being and older people: A review of the literature. Canadian Journal of Occupational Therapy, 70, 51-59.

Teixeira, C. y Kalinoski, S. (2003). La importancia del deporte como factor social en las matrículas en escuelas deportivas de la administración pública de Pindamonhangaba, Brasil. Recuperado de http:// www.efdeportes.com.

Terol, M., López, S., Rodríguez, J., Pastor, M., Mora, M., Martín, Leyda, J., Neipp, M. y Lizón, J. (2000). Diferencias en la calidad de vida: Un estudio longitudinal de pacientes de cáncer recibiendo tratamiento de quimioterapia. Anales de Psicología, 16, 111-122.

Terreros, J., Arnaudas, C. y Cucullo, J. (1992) Estudio médico-deportivo en la tercera edad. Valoración médica. Apunts, 112, 115-125.

Van Amersfoort, Y. (1996). Prescripción de ejercicio físico y salud mental. En J. Serra Grima (ed.), Prescripción de ejercicio físico para la salud (pp. 303-339). Barcelona: Paidotribo.

Villar, F., Triadó, C., Solé, C. y Osuna, M. (2003). Bienestar, adaptación y envejecimiento: cuando la estabilidad significa cambio. Revista Multidisciplinar de Gerontología, 13, 152-162.

Vuori, I. (2004). Physical inactivity is a cause and physical activity is a remedy for major public health problems. Kinesiology, 36(2), 123-153.

Walsh, R. (2011). Lifestyle and Mental Health. American Psychologist, 66(7), 579-592.

Weinberger, M., Greene, J. y Mamlin J. (1981). Health beliefs and smoking behavior. American Journal Public Health, 71, 1253-1255.

Weinstein, N. (1988). The precaution adoption process. Health Psychol, 7, 355-386.

World Health Organization. (2008). Benefits of physical activity. Recuperado de http://www.who.int/ dietphysicalactivity/factsheet_benefits/en/print.html.

Yanguas, J. (2006): Análisis de la calidad de vida relacionada con la salud en la vejez desde una perspectiva multidimensional. Madrid: Imserso. 